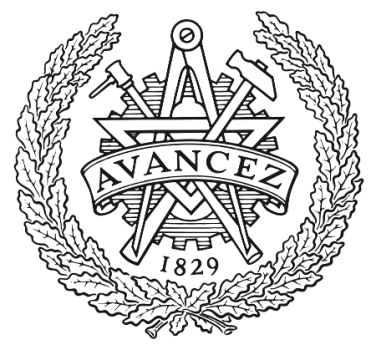

CHALMERS

UNIVERSITY OF TECHNOLOGY

\title{
Auto-Calibration of Co-located Uniform Linear Array Antennas
}

Downloaded from: https://research.chalmers.se, 2023-04-26 13:20 UTC

Citation for the original published paper (version of record):

McKelvey, T. (2019). Auto-Calibration of Co-located Uniform Linear Array Antennas. 2019 IEEE 8th International Workshop on Computational Advances in Multi-Sensor Adaptive Processing, CAMSAP 2019 - Proceedings: 56-60. http://dx.doi.org/10.1109/CAMSAP45676.2019.9022483

N.B. When citing this work, cite the original published paper. 


\title{
AUTO-CALIBRATION OF CO-LOCATED UNIFORM LINEAR ARRAY ANTENNAS
}

\author{
Tomas McKelvey \\ Electrical Engineering \\ Chalmers University of Technology \\ Gothenburg, Sweden
}

\begin{abstract}
An algorithm for auto-calibration of a group of co-located uniform linear array antennas is presented. If the number of signal sources are known and, for at least one array, the ratio of the gains between two consecutive antenna elements is known, the individual unknown antenna gains can be estimated. The method is based on determining the antenna calibration parameters such that a matrix built from the array snapshots has a given rank. A numerical example illustrates the performance of the method. The numerical results suggest that the method is consistent in SNR.
\end{abstract}

Index Terms - Estimation, Optimization, Calibration, Linear antenna arrays, Direction-of-arrival estimation

\section{INTRODUCTION}

In high performing radar systems calibration is necessary to overcome the inevitable deviations that is the result of variations in the manufacturing. In this contribution we consider the problem of calibrating a group of co-located array antennas, each with a uniform linear configuration. For co-located arrays we assume all arrays are sensing the same directions to the signal sources.

The problem of estimating the unknown gains in a linear array in a blind fashion, i.e. without knowing the directions to the active signal sources is called auto-calibration. We can also regard this as a joint estimation of the array gain and the directions to the signal sources. This problem has been treated by many authors over the years $[1,2,3,4,5,6,7,8]$ with various assumptions on the array and properties of the signal sources sensed by the arrays.

In this contribution we assume the number of signal sources are known. We describe an auto-calibration method which is based on the low-rank properties of a matrix built from Hankel matrices with snapshot data from all the arrays. This matrix is explored in Kung's subspace method [9] for direction of arrival (DOA) estimation. The Hankel matrices are parametrized with the unknown calibration parameters for the different arrays. The calibration parameters are determined by minimizing a cost function involving the rank of the constructed matrix. If we assume, at least for one array, the complex ratio between the true gain of two consecutive antenna elements is known, both the direction of arrivals and the

This research has been carried out in the ChaseOn Centre in a project financed by Vinnova, Chalmers, Food Radar Systems, Keysight Technologies, Medfield Diagnostics, Saab and UniqueSec. gain of all the antenna array elements can be recovered. The contribution of this paper is to extend the method presented in [10] to the case of a group of co-located ULAs.

The paper is outlined as follows. After the problem formulation in we present some system theory results in Section 2. The calibration method is outlined in Section 3 and numerical illustrations are given in Section 4.

\subsection{Notation}

By $(\cdot)^{T}$ and $(\cdot)^{*}$ we denote the transpose and the Hermitian transpose respectively. The Hadamard product $\odot$ is the element wise matrix product, i.e. $[\mathbf{A} \odot \mathbf{B}]_{i j}=[\mathbf{A}]_{i j}[\mathbf{B}]_{i j}$. For column vectors $\mathbf{a}$ and $\mathbf{b}$, we have $\mathbf{a} \odot \mathbf{b}=\operatorname{diag}(\mathbf{a}) \mathbf{b}$, where $\operatorname{diag}(\mathbf{a})$ is a diagonal matrix with the elements in vector $\mathbf{a}$ on the diagonal.

\subsection{Problem Formulation}

We consider the standard formulation by assuming $P$ signal sources are emitting narrowband signals which are sensed by $N_{a}$ different uniform linear arrays (ULAs), each one with $M$ antenna elements. After IQ demodulation the measured vector signal at snapshot $n$ at the array $a$ can be modeled as [11]

$$
\tilde{\mathbf{y}}_{a}(n)=\mathbf{y}_{a}(n)+\mathbf{v}_{a}(n)=\sum_{p=1}^{P} \mathbf{a}_{a}\left(\theta_{p}\right) x_{a, p}(n)+\mathbf{v}_{a}(n)
$$

where $\theta_{p}$ is the direction of the arrival for signal source $p$, $\mathbf{a}_{a}\left(\theta_{p}\right) \in \mathbb{C}^{M}$ is the steering vector, $x_{a, p}(n)$ is the complex amplitude for signal source $p$, and $\mathbf{v}_{a}(n)$ is an assumed additive noise. Here $\mathbf{y}_{a}(n)$ denotes the noise free snapshot vector. In the derivation of the method below we assume noise free data and will return to the more relevant case with noise present in the numerical evaluation of the calibration method.

Due to manufacturing inaccuracies and electromagnetic effects the array steering vector $\mathbf{a}_{a}\left(\theta_{p}\right)$ is expressed as

$$
\mathbf{a}_{a}\left(\theta_{p}\right)=\mathbf{g}_{a} \odot \mathbf{a}_{0}\left(\theta_{p}\right)
$$

where $\mathbf{a}_{0}\left(\theta_{p}\right)$ is the ideal steering vector and

$$
\mathbf{g}_{a}=\left[g_{a, 1}, g_{a, 2}, \ldots, g_{a, M}\right]^{T} \in \mathbb{C}^{M}
$$

is the static array gain for array $a$ and we assume $g_{a, i} \neq 0$ for all $i$. The problem we address in this paper is how to jointly determine the unknown antenna gains and the direction of arrivals given snapshot data from all the co-located arrays. 


\subsection{Uniform linear array}

Each antenna array is assumed to have a linear shape and equal spacing $\Delta$ between the antenna elements. Assume signal source $p$ is located at a direction $\theta_{p}$ relative to the direction perpendicular to the extent of the antenna array. Define the spatial frequency $\omega_{p} \triangleq \frac{2 \pi \Delta}{\lambda} \sin \theta_{p}$, where $\lambda$ is the wavelength of the incoming signal. The steering vector for signal source $p$ can then be described as

$$
\mathbf{a}_{0}\left(\theta_{p}\right)=\left[\begin{array}{lllll}
1 & e^{j \omega_{p}} & e^{j 2 \omega_{p}} & \ldots & e^{j(M-1) \omega_{p}}
\end{array}\right]^{T} .
$$

With the definitions

$$
\mathbf{A} \triangleq \operatorname{diag}\left(\left[e^{j \omega_{1}}, e^{j \omega_{2}}, \ldots, e^{j \omega_{P}}\right]\right), \quad \mathbf{c} \triangleq\left[\begin{array}{lll}
1 & \ldots & 1
\end{array}\right]
$$

and

$$
\mathbf{x}_{a}(n) \triangleq\left[x_{a, 1}(n) \quad x_{a, 2}(n) \quad \ldots \quad x_{a, P}(n)\right]^{T}
$$

we obtain for antenna $a$ and element at position $m$

$$
y_{a, m}(n)=g_{a, m} \mathbf{c} \mathbf{A}^{m-1} \mathbf{x}_{a}(n)
$$

By collecting the antenna responses from the $N$ snapshots at element $m$ we have

$$
\begin{aligned}
\mathbf{y}_{a, m} & \triangleq\left[\begin{array}{llll}
y_{a, m}(1) & y_{a, m}(2) & \cdots & y_{a, m}(N)
\end{array}\right] \\
& =g_{a, m} \mathbf{c} \mathbf{A}^{m-1} \mathbf{X}_{a}
\end{aligned}
$$

where

$$
\mathbf{X}_{a} \triangleq\left[\begin{array}{llll}
\mathbf{x}_{a}(1) & \mathbf{x}_{a}(2) & \cdots & \mathbf{x}_{a}(N)
\end{array}\right]
$$

We note that if $g_{a, m}=1$ for all $m$ then $\mathbf{y}_{a, m}$ can be seen as the Markov parameters for a linear system described by the triple $\left(\mathbf{A}, \mathbf{X}_{a}, \mathbf{c}\right)$. We will rely on this fact in the derivation that follows. Finally we note that antenna gain $\mathbf{g}_{a}$ and the signal amplitude matrix $\mathbf{X}_{a}$ in the model (4) cannot be uniquely separated as an arbitrary non-zero complex scalar value can be moved between them with identical $\mathbf{y}_{a, m}$. Here we fix $g_{a, 1}=1$ to remove this ambiguity.

\section{PRELIMINARIES}

\subsection{Some results from systems theory}

Before proceeding we recall some well known results from linear systems theory [12]. We will here discuss properties of the matrix triple $\left(\mathbf{A}, \mathbf{X}_{a}, \mathbf{c}\right)$, where $\mathbf{A} \in \mathbb{C}^{P \times P}, \mathbf{c} \in \mathbb{C}^{1 \times P}$, and $\mathbf{X}_{a} \in \mathbb{C}^{P \times N}$ defining a sequence $\mathbf{y}_{a, m} \triangleq \mathbf{c} \mathbf{A}^{m-1} \mathbf{X}_{a} \in$ $\mathbb{C}^{1 \times N}$ for all $m=1,2, \ldots$ The triple $\left(\mathbf{A}, \mathbf{X}_{a}, \mathbf{c}\right)$ is called a realization of order $P$ of the sequence $\left\{\mathbf{y}_{a, m}\right\}_{m=1}^{\infty}$. The extended controllability matrix of order $r$ is

$$
\mathcal{C}_{r}\left(\mathbf{A}, \mathbf{X}_{a}\right) \triangleq\left[\begin{array}{llll}
\mathbf{X}_{a} & \mathbf{A} \mathbf{X}_{a} & \cdots & \mathbf{A}^{r-1} \mathbf{X}_{a}
\end{array}\right]
$$

and the extended observability matrix of order $s$ is

$$
\mathcal{O}_{s}(\mathbf{A}, \mathbf{c}) \triangleq\left[\begin{array}{llll}
\mathbf{c}^{T} & (\mathbf{c} \mathbf{A})^{T} & \cdots & \left(\mathbf{c} \mathbf{A}^{s-1}\right)^{T}
\end{array}\right]^{T}
$$

A realization of the sequence $\left\{\mathbf{y}_{a, m}\right\}_{m=1}^{\infty}$ is minimal if there exists no other realization of the sequence that has a lower order. The following result is instrumental and can for instance be found in linear systems theory literature e.g. [13, 12].
Lemma 1 A realization $\left(\mathbf{A}, \mathbf{X}_{a}, \mathbf{c}\right)$ of order $P$ is minimal if and only if $\operatorname{rank} \mathcal{C}_{P}\left(\mathbf{A}, \mathbf{X}_{a}\right)=\operatorname{rank} \mathcal{O}_{P}(\mathbf{A}, \mathbf{c})=P$.

Trivially we note that $\operatorname{rank} \mathcal{O}_{s}(\mathbf{A}, \mathbf{C})=P$ for all $s>P$ if $\operatorname{rank} \mathcal{O}_{P}(\mathbf{A}, \mathbf{c})=P$. Also, by the Cayley-Hamilton theorem, if $\operatorname{rank} \mathcal{O}_{s}(\mathbf{A}, \mathbf{c})=P$ for some $s>P$ then $\operatorname{rank} \mathcal{O}_{P}(\mathbf{A}, \mathbf{C})=P$. Dual results hold for the controllability matrix.

If we arrange the first $s+r-1$ samples in the sequence $\mathbf{y}_{a, m}$ as a block Hankel matrix we obtain

$$
\mathbf{Y}_{a, s, r}=\left[\begin{array}{cccc}
\mathbf{y}_{a, 1} & \mathbf{y}_{a, 2} & \cdots & \mathbf{y}_{a, r} \\
\mathbf{y}_{a, 2} & \mathbf{y}_{a, 3} & \cdots & \mathbf{y}_{a, r+2} \\
\vdots & \vdots & \vdots & \vdots \\
\mathbf{y}_{a, s} & \mathbf{y}_{a, s+1} & \cdots & \mathbf{y}_{a, s+r-1}
\end{array}\right] \in \mathbb{C}^{s \times r N}
$$

The Hankel structure implies that the obtained matrix has the same block elements on all anti-diagonals. The following result ties together the minimality of a realization and the rank properties of the associated Hankel matrix, which can be found in e.g. $[13,14]$. A compact full proof of the result below can be found in [10].

Theorem 1 Consider a sequence of row vectors $\left\{\mathbf{y}_{a, m}\right\}_{m=1}^{2 P}$ and the corresponding block Hankel matrix $\mathbf{Y}_{a, s, r}$ defined in (9). Then $\operatorname{rank} \mathbf{Y}_{a, P+1, P+1}=n$ and $\operatorname{rank} \mathbf{Y}_{a, P, P+1}=P$ if and only if there exists a minimal realization $\left(\mathbf{A}, \mathbf{X}_{a}, \mathbf{c}\right)$ of order $P$ such that $\mathbf{y}_{a, m}=\mathbf{c A}^{m-1} \mathbf{X}_{A}$ for $m=1, \ldots, 2 P$.

Theorem 2 Assume the array a is ideal, $\mathbf{g}_{a}^{T}=[1, \ldots, 1]$, the $P$ signal sources have distinct spatial frequencies $\omega_{p}$ and that for at least one snapshot $n^{\prime}$ each signal source $p, x_{a, p}\left(n^{\prime}\right)$ is non-zero. Then the realization $\left(\mathbf{A}, \mathbf{X}_{a}, \mathbf{c}\right)$ given by (2) and (6) is minimal and $\operatorname{rank} \mathbf{Y}_{a, P+1, P+1}=\operatorname{rank} \mathbf{Y}_{a, P, P+1}=P$.

Proof: See [10].

Finally we give the following key result.

Theorem 3 Define

$$
\mathbf{H}_{s, r} \triangleq\left[\begin{array}{llll}
\mathbf{Y}_{1, s, r} & \mathbf{Y}_{2, s, r} & \cdots & \mathbf{Y}_{N_{a}, s, r}
\end{array}\right]
$$

as the matrix formed from the Hankel matrices from the individual arrays. Assume the conditions given in Theorem 2 hold for all the $N_{a}$ arrays. Then

$$
\operatorname{rank} \mathbf{H}_{P+1, P+1}=\operatorname{rank} \mathbf{H}_{P, P+1}=P
$$

Proof: The range space for $\mathbf{Y}_{a, s, r}$ is $\mathcal{O}_{s}(\mathbf{A}, \mathbf{c})$ and is hence the same for all arrays which imply that $\operatorname{rank} \mathbf{H}_{s, r}$ is upper bounded by $P$ for $s, r \geq P$. That the lower bound for rank $\mathbf{H}_{s, r}$ is $P$ follows directly from Theorem 2 whenever $s, r \geq P$.

The result shows that if all the arrays are calibrated the Hankel matrix $\mathbf{H}_{P+1, P+1}$ will be of $\operatorname{rank} P$. This is the starting point for the calibration algorithm we describe in the next section. 


\section{AUTO-CALIBRATION}

We will now introduce the calibration method where we consider two approaches. The first approach is simply to treat each array individually and calibrate it according to the method presented in [10]. We denote this method Autocalibration indiv. The second method, which is the topic of this paper, is to estimate the calibration parameters jointly for all $N_{a}$ arrays simultaneously and thereby benefit in performance since we explicitly utilize that all the arrays sense the same $P$ signal sources. We denote this method Autocalibration joint.

The calibrated output for array $a$ is defined by

$$
\mathbf{y}_{a, m}^{c} \triangleq h_{a, m} \mathbf{y}_{a, m}, \quad m=1, \ldots, M
$$

where $h_{a, m} \in \mathbb{C}$ compensates for the deviations from the ideal unit antenna gain. (Note that optimal calibration for array $a$ implies $h_{a, m} g_{a, m}=1$ for $m=1, \ldots, M$.) Given $\mathbf{h}_{a} \triangleq\left[h_{a, 1}, \ldots, h_{a, M}\right]$ and the snapshot data, define the calibrated Hankel matrix

$$
\mathbf{Y}_{a, s, r}^{c}\left(\mathbf{h}_{a}\right) \triangleq\left[\begin{array}{cccc}
\mathbf{y}_{a, 1}^{c} & \mathbf{y}_{a, 2}^{c} & \cdots & \mathbf{y}_{a, r}^{c} \\
\mathbf{y}_{a, 2}^{c} & \mathbf{y}_{a, 3}^{c} & \cdots & \mathbf{y}_{a, r+2}^{c} \\
\vdots & \vdots & \vdots & \vdots \\
\mathbf{y}_{a, s}^{c} & \mathbf{y}_{a, s+1}^{c} & \cdots & \mathbf{y}_{a, s+r-1}^{c}
\end{array}\right] \in \mathbb{C}^{s \times r N}
$$

By assumption $g_{a, 1}=1$ so we fix $h_{a, 1}=1$. Let $\mathbf{h}^{T} \triangleq$ $\left[\begin{array}{lll}\mathbf{h}_{1}^{T} & \ldots & \mathbf{h}_{N_{A}}^{T}\end{array}\right]$ be the vector collecting all calibration parameters and define

$\mathbf{H}_{s, r}^{c}(\mathbf{h}) \triangleq\left[\begin{array}{llll}\mathbf{Y}_{1, s, r}^{c}\left(\mathbf{h}_{1}\right) & \mathbf{Y}_{2, s, r}^{c}\left(\mathbf{h}_{2}\right) & \ldots & \mathbf{Y}_{N_{a}, s, r}^{c}\left(\mathbf{h}_{N_{a}}\right)\end{array}\right]$

By Theorem 3 it is clear that if $h_{a, m} g_{a, m}=1$ for $m=$ $1, \ldots, M$ and $a=1, \ldots, N_{a}$, all arrays are correctly calibrated, $\operatorname{rank} \mathbf{H}_{P+1, P+1}^{c}=\operatorname{rank} \mathbf{H}_{P, P+1}^{c}=P$ and the individual array response are given by a realizations $\left(\hat{\mathbf{A}}, \hat{\mathbf{X}}_{a}, \hat{\mathbf{c}}\right)$. Furthermore, the eigenvalues of $\hat{\mathbf{A}}$ are the same as $\mathbf{A}$ in (2) as the matrices are similar. From the eigenvalues the DOAs to the $P$ signal sources can be recovered.

The proposed auto-calibration method proceeds by finding a calibration vector $\mathbf{h}$ that makes the rank of the calibrated matrices $\mathbf{H}_{P+1, P+1}^{c}(\mathbf{h})$ and $\mathbf{H}_{P, P+1}^{c}(\mathbf{h})$ equal to $P$. A vector h which make the matrices have rank $P$ does not directly imply that the correct calibration parameters have been found. The following result show two possible cases of ambiguity.

Theorem 4 Consider the scenario in Theorem 2 but with an arbitrary non-zero antenna gain for all array elements. If, for each array, the calibration vector has either of the following two forms:

$$
\begin{aligned}
& \text { 1. } \mathbf{h}_{a}^{T}=\left[\begin{array}{lllll}
1 & g_{a, 2}^{-1} \beta & g_{a, 3}^{-1} \beta^{2} & \cdots & g_{a, M}^{-1} \beta^{M-1}
\end{array}\right] \text { where } \\
& \beta \in \mathbb{C} \text { and } \beta \neq 0 \text { is arbitrary. } \\
& \text { 2. } \mathbf{h}_{a}^{T}=\left[\begin{array}{lllll}
h_{a, 1}^{\prime} & h_{a, 2}^{\prime} & \cdots & h_{a, P}^{\prime} & 0 \cdots 0
\end{array}\right] \text { where } h_{a, P}^{\prime} \mathbf{y}_{a, P} \neq \\
& 0 \text { and } h_{a, m}^{\prime} \in \mathbb{C} \text { for } m=1, \ldots, P-1 \text { are arbitrary. }
\end{aligned}
$$

then $\operatorname{rank} \mathbf{H}_{P+1, P+1}^{c}(\mathbf{h})=\operatorname{rank} \mathbf{H}_{P, P+1}^{c}(\mathbf{h})=P$. The system matrix $\hat{\mathbf{A}}$ for the corresponding realizations will have eigenvalues:

$$
\begin{aligned}
& \text { 1. } \lambda_{i}=\beta e^{j \omega_{i}} \text { for } i=1, \ldots, P \\
& \text { 2. } \lambda_{i}=0 \text { for } i=1, \ldots, P .
\end{aligned}
$$

Proof: See [10] .

The theorem gives sufficient conditions on the calibration vector $h_{a, m}$ such that the set of equations

$h_{a, m} \mathbf{y}_{a, m}=h_{a, m} \mathbf{c} \mathbf{A}^{m-1} \mathbf{X}_{a}=\tilde{\mathbf{c}} \tilde{\mathbf{A}}^{m-1} \tilde{\mathbf{X}}_{a}, \quad m=1, \ldots, M$

has a solution, where $\left(\tilde{\mathbf{A}}, \tilde{\mathbf{X}}_{a}, \tilde{\mathbf{c}}\right)$ is a minimal realization of dimension $P$. If the ratio between the true gain of two consecutive antenna elements are known for at least one of the arrays, the unknown scalar $\beta$ in Theorem 4 Case 1 can be resolved. This necessary knowledge for identifiability is similar to when instead a direction to one reference source is known [1]. Without loss of generality assume the gain ratio is given by $\gamma_{a}=g_{a, 2} / g_{a, 1}=g_{a, 2}$ since $g_{a, 1}=1$. Hence we obtain that $\beta=h_{a, 2} \gamma_{a}$. With this information the elements of the calibration vector is modified as $\hat{h}_{a, m}=h_{a, m} \beta^{1-m}$, $m=2, \ldots, M$, removing the ambiguity. If the gain ratio is known for several arrays, the estimate of $\beta$ can be improved by averaging the result from each array.

\subsection{The calibration algorithm}

Based in the properties derived in the previous section the desired calibration vector $\mathbf{h}$ can be found as the solution to the following optimization problem:

$$
\begin{aligned}
& \min _{\mathbf{h}, \mathbf{L}}\left\|\mathbf{L}-\mathbf{H}_{P+1, P+1}^{c}(\mathbf{h})\right\|_{F}^{2} \\
& \text { s.t. } \quad \operatorname{rank} \mathbf{L}=P, \quad h_{a, 1}=1, \quad a=1,2, \ldots, N_{a}
\end{aligned}
$$

This problem is NP-hard due to combination of the rank constraint and the structural properties of $\mathbf{H}_{P+1, P+1}^{c}(\mathbf{h})$. A possible remedy could be using a relaxation method, e.g. the nuclear norm, [15] could be used as a proxy for the rank constraint. A complication with the nuclear norm relaxation is that an extra hyperparameter needs to be determined in order to obtain the correct rank $P$. Here we employ a heuristic method outlined in Algorithm 1 for solving (16) that does not involve searching over additional parameters. The method iterate between solving for $\mathbf{L}$ and $\mathbf{h}$.

\section{Algorithm 1}

1. Initialize $\mathbf{h}=[1,1, \ldots, 1]$

2. Determine the $S V D$

$$
\mathbf{H}_{P+1, P+1}^{c}(\mathbf{h})=\left[\mathbf{U}_{1} \mathbf{u}_{2}\right]\left[\begin{array}{ccc}
\mathbf{S} & 0 & 0 \\
0 & \sigma_{P+1} & 0
\end{array}\right]\left[\begin{array}{c}
\mathbf{V}_{1}^{*} \\
\mathbf{V}_{2}^{*} \\
\mathbf{V}_{3}^{*}
\end{array}\right]
$$

with $\mathbf{S}=\operatorname{diag}\left(\sigma_{1}, \ldots, \sigma_{P}\right) \in \mathbb{R}^{P \times P}$ where $\sigma_{1} \geq$ $\ldots \geq \sigma_{P} \geq \sigma_{P+1}$ are the singular values and set $\mathbf{L}=\overline{\mathbf{U}}_{1} \mathbf{S V}_{1}^{*}$.

3. Solve the least-squares problem

$$
\min _{\mathbf{h}}\left\|\mathbf{L}-\mathbf{H}_{P+1, P+1}^{c}(\mathbf{h})\right\|_{F}^{2}
$$

and for each array a scale $\mathbf{h}_{a}:=\mathbf{h}_{a} / h_{a, 1}$. 


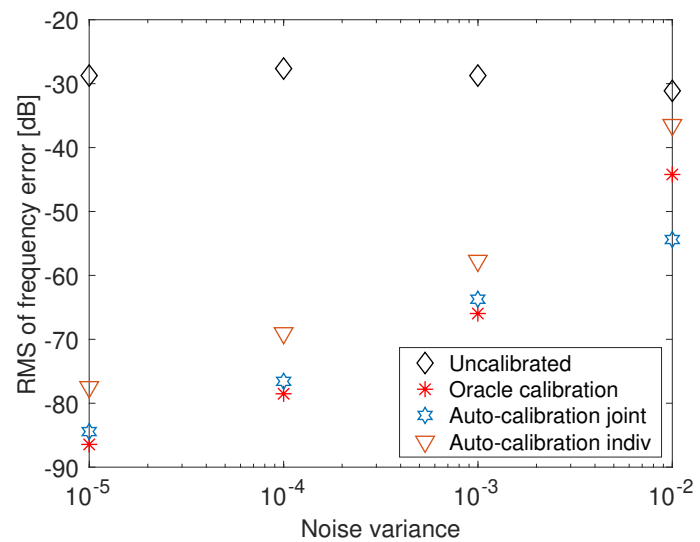

Fig. 1. RMS error for estimated spatial frequencies versus variance of the noise.

4. Repeat 2-3 until the $\sigma_{P+1} / \sigma_{P}$ is below some set threshold.

5. Set $\beta=\frac{1}{N_{a}^{\prime}} \sum_{k=1}^{N_{a}^{\prime}} h_{a_{k}, 2} \gamma_{a_{k}}$ and adjust the calibration vector $\hat{h}_{a, m}=h_{a, m} \beta^{1-m}, m=2, \ldots, M, a=$ $1, \ldots, N_{a}$. Here the index set $\left\{a_{1}, \ldots, a_{N_{a}^{\prime}}\right\}$ contain the indices to the $N_{a}^{\prime}$ arrays where the gain ratio $\gamma_{a}$ is known.

If a solution to the original problem (16) has been found, for the noise free case, then $\operatorname{rank} \mathbf{H}_{P+1, P+1}^{c}(\mathbf{h})=P$ and hence $\sigma_{P+1}=0$. This property is the basis for the stopping criteria in Step 5). In the noisy case $\sigma_{P+1} / \sigma_{P}>0$ also for the correct calibration. The signal to noise ratio will determine a suitable threshold for termination of the algorithm.

The calibrated array responses derived according to Algorithm 1 can be used to perform an estimate of the directions of arrival with an arbitrary DOA estimation algorithm, e.g. maximum-likelihood, Kung's method or ESPRIT [16, 9, 17]. All methods allow to use the snapshots for all arrays to yield one estimate of the DOAs. In Kung's method this is accomplished by estimating the range space of the matrix $\mathbf{H}_{P+1, P+1}^{c}(\mathbf{h})$. This estimate is delivered by a (final) SVD according to (17) where the matrix $\mathbf{U}_{1}$ is the estimate of the desired range space.

\section{NUMERICAL ILLUSTRATION}

A Monte Carlo simulation is used to illustrate the performance in a scenario with $N_{a}=10$ uniform linear arrays each with $M=16$ elements and two signal sources with relative spatial frequencies $\omega_{1}=-2 \pi \cdot 0.122$ and $\omega_{2}=$ $2 \pi \cdot 0.22$. Array responses from $N=10$ snapshots are generated for each array. A zero mean complex circularly symmetric Gaussian distributed noise with variance ranging from $10^{-4}$ to $10^{-1}$ is added to the noise free array responses. For each Monte Carlo run and for each array an individual antenna gain vector is generated by adding, to the ideal unit gain, a zero mean complex circularly symmetric uniformly distributed random variable with variance 0.2 . The performance is evaluated by generating 100 independent realizations of the source signals, noise, and the antenna gains. Al-

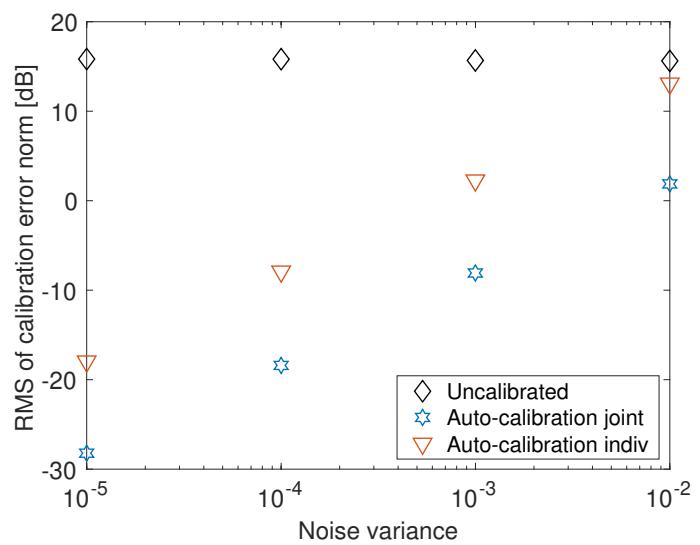

Fig. 2. RMS of calibration error norm versus variance of the noise.

gorithm 1 is used to estimate the unknown calibration vector using the information $\gamma_{a}=g_{a, 2} / g_{a, 1}$ for all arrays. This calibration is denoted Auto-calibration joint. Algorithm 1 is used for each array separately, i.e. the calibration is determined without using the measurements from the other arrays but using the information $\gamma_{a}=g_{a, 2} / g_{a, 1}$. This corresponds to the algorithm presented in [10]. This calibration is denoted Autocalibration indiv. Kung's algorithm [9] is used to estimate the two unknown spatial frequencies using the auto-calibrated data for the two different calibration methods. These estimates are compared with an oracle calibration where we employ Kung's algorithm to the array data with the correct calibration vector. Finally we also compare with the estimate obtained from Kung's algorithm by directly using the uncalibrated raw data.

The results of the numerical evaluation are reported in figures 1-2. In Figure 1 the root mean square (RMS) errors for the spatial frequencies for the four cases are compared. We notice that the auto-calibrated case where each array is calibrated individually improves the performance compared to the uncalibrated case. As expected performing a joint calibration improves the performance significantly but is inferior to the oracle based estimate. In Figure 2 the RMS of the norm of the calibration error is illustrated. The joint calibration is superior to the individual calibration. In both figures we see that the errors decrease with improved SNR which suggests that the method is consistent in SNR.

\section{SUMMARY}

In this contribution we have presented an algorithm which can be used to calibrate a group of co-located ULAs without full knowledge of the environment. Particularly, if the number of signal sources and the gain ratio between two consecutive antenna elements for one array are known we have shown that we can determine the individual unknown antenna gains. The numerical results sugest that the proposed method is consistent in SNR. 


\section{REFERENCES}

[1] A Paulraj and T Kailath, "Direction of arrival estimation by eigenstructure methods with unknown sensor gain and phase," in ICASSP '85. IEEE International Conference on Acoustics, Speech, and Signal Processing, apr 1985, vol. 10, pp. 640-643.

[2] Jungtai Kim, Hyun Jong Yang, Byung Wook Jung, and Joohwan Chun, "Blind calibration for a linear array with gain and phase error using independent component analysis," IEEE Antennas and Wireless Propagation Letters, vol. 9, pp. 1259-1262, 2010.

[3] Peter M Schultheiss and John P Ianniello, "Optimum range and bearing estimation with randomly perturbed arrays," The Journal of the Acoustical Society of America, vol. 68, no. 1, pp. 167-173, 1980.

[4] Anthony J Weiss and Benjamin Friedlander, "Eigenstructure methods for direction finding with sensor gain and phase uncertainties," Circuits, Systems and Signal Processing, vol. 9, no. 3, pp. 271-300, sep 1990.

[5] M Viberg and A L Swindlehurst, "A Bayesian approach to auto-calibration for parametric array signal processing," IEEE Transactions on Signal Processing, vol. 42, no. 12 , pp. 3495-3507, dec 1994.

[6] D Astely, A L Swindlehurst, and B Ottersten, "Spatial signature estimation for uniform linear arrays with unknown receiver gains and phases," IEEE Transactions on Signal Processing, vol. 47, no. 8, pp. 2128-2138, aug 1999.

[7] K N Ramamohan, S Prabhakar Chepuri, D F Comesaña, and G Leus, "Blind Calibration of Sparse Arrays for DOA Estimation with Analog and One-bit Measurements," in ICASSP 2019 - 2019 IEEE International Conference on Acoustics, Speech and Signal Processing (ICASSP), may 2019, pp. 4185-4189.

[8] Krishnaprasad Nambur Ramamohan, Sundeep Prabhakar Chepuri, Daniel Fernandez Comesana, Graciano Carrillo Pousa, and Geert Leus, "Blind Calibration for Acoustic Vector Sensor Arrays," ICASSP, IEEE International Conference on Acoustics, Speech and Signal Processing - Proceedings, vol. 2018-April, pp. 3544-3548, 2018.

[9] S Y Kung, "A New Identification and Model Reduction Algorithm via Singular Value Decomposition," in Proc. 12th Asilomar Conference on Circuits, Systems and Computers, Pacific Grove, CA, 1978, pp. 705-714.

[10] T McKelvey, "Auto-calibration of uniform linear array antennas," in Proc. 27th European Signal Processing Conference, A Coruña, Spain, Sept. 2019, EURASIP.

[11] H Krim and M Viberg, "Two decades of array signal processing research: the parametric approach," IEEE Signal Processing Magazine, vol. 13, no. 4, pp. 67-94, jul 1996.
[12] T Kailath, Linear Systems, Prentice-Hall, Englewood Cliffs, New Jersey, 1980.

[13] Eduardo D Sontag, Mathematical control theory: Deterministic finite dimensional systems, Springer, 2 edition, 1998.

[14] A A H Damen, R P Guidorzi, A K Hajdasinski, and P M J den Hof, "On multivariable partial realization," Int. J. Control, vol. 41, no. 3, pp. 589-613, 1985.

[15] M Fazel, T Pong, D Sun, and P Tseng, "Hankel Matrix Rank Minimization with Applications to System Identification and Realization," SIAM Journal on Matrix Analysis and Applications, vol. 34, no. 3, pp. 946-977, 2013.

[16] P Stoica and R Moses, Introduction to Spectral Analysis, Prentice-Hall, Englewood Cliffs, USA, 1997.

[17] R. Roy and T. Kailath, "ESPRIT - Estimation of Signal Parameters via Rotational Invariance Techniques," IEEE Trans. on Acoustics, Speech and Signal Processing, vol. 37, no. 7, pp. 984-995, jul 1989. 